\title{
The Knowledge and Awareness of the General Public About Obstructive Sleep Apnea
}

\author{
Leeba Rezaie ${ }^{1}$ and Behnam Khaledi-Paveh ${ }^{1,2,{ }^{*}}$ \\ ${ }^{1}$ Sleep Disorders Research Center, Kermanshah University of Medical Sciences, Kermanshah, Iran \\ ${ }^{2}$ Department of Psychiatric Nursing, School of Nursing And Midwifery, Kermanshah University of Medical Sciences, Kermanshah, Iran \\ "Corresponding author: Farabi Hospital, Dowlatabad Blvd, Kermanshah, Iran. Tel: +98-9183310752, Fax: +98-8338264163, Email: bkhaledi@ymail.com
}

Received 2018 April 21; Accepted 2018 May 23.

Keywords: Obstructive Sleep Apnea, Disorder, General Public

\section{Dear Editor,}

Obstructive sleep apnea (OSA) is a common sleep disorder in the general public that is characterized by recurrent collapse of the upper airway that leads to apnea/hypopnea during sleep and repetitive arousal. It is also associated with daytime symptoms such as fatigue and sleepiness and an increased risk of cardiovascular diseases, hypertension and stroke and thereby an increased risk of mortality (1). The early diagnosis and treatment of patients with OSA is therefore necessary.

Despite its high prevalence, OSA remains an underdiagnosed condition, and some studies have reported that up to $90 \%$ of people with moderate to severe OSA do not get a proper diagnosis by a physician (2). It seems that the knowledge and awareness of the general public about this condition is poor.

In a study to follow up patients diagnosed with OSA at the Sleep Disorders Research Center of Kermanshah University of Medical Sciences, we reviewed the medical records of 101 patients with moderate to severe OSA. These included symptomatic patients with an apnea/hypopnea index (AHI) $\geq 5$ as well as non-symptomatic patients with AHI $\geq 15$ based on their polysomnography results (3). In these cases, the patients should undergo special treatments such as continuous positive airway pressure (CPAP) (4). In this review study, except for three cases $(<3 \%)$, who were themselves physician, all the patients were unaware of their symptoms and condition. They were referred by physicians for sleep assessment while following up on their other diseases. All of the patients stated that they knew nothing about OSA and its symptoms, although they had experienced it for a long time.

Although this result cannot be generalized to all patients with OSA, including those diagnosed with mild OSA or those seeking a proper diagnosis, it is important to note that, because of the general lack of knowledge about this condition, people are at risk for adverse consequences such as cardiovascular diseases and hypertension, which can impose a heavy cost on the health system. Promoting awareness about this condition in the general public is thus necessary. To meet this purpose, a study needs to be designed that can assess the knowledge and awareness of the general public about OSA using a valid questionnaire. The results of such a study can offer comprehensive information and help plan appropriate interventions for promoting the knowledge and awareness of the public about OSA.

\section{References}

1. Khazaie H, Najafi F, Rezaie L, Tahmasian M, Sepehry AA, Herth FJ. Prevalence of symptoms and risk of obstructive sleep apnea syndrome in the general population. Arch Iran Med. 2011;14(5):335-8. [PubMed: 21888458].

2. Redline S, Sotres-Alvarez D, Loredo J, Hall M, Patel SR, Ramos A, et al. Sleep-disordered breathing in Hispanic/Latino individuals of diverse backgrounds. The Hispanic Community Health Study/Study of Latinos. Am J Respir Crit Care Med. 2014;189(3):335-44. doi: 10.1164/rccm.201309-1735OC. [PubMed: 24392863]. [PubMed Central: PMC3977733].

3. Epstein LJ, Kristo D, Strollo PJ Jr, Friedman N, Malhotra A, Patil $\mathrm{SP}$, et al. Clinical guideline for the evaluation, management and long-term care of obstructive sleep apnea in adults. J Clin Sleep Med. 2009;5(3):263-76. [PubMed: 19960649]. [PubMed Central: PMC2699173].

4. Montesi SB, Edwards BA, Malhotra A, Bakker JP. The effect of continuous positive airway pressure treatment on blood pressure: a systematic review and meta-analysis of randomized controlled trials. J Clin Sleep Med. 2012;8(5):587-96. doi: 10.5664/jcsm.2170. [PubMed: 23066375]. [PubMed Central: PMC3459209]. 